\title{
Probability Grid: A Location Estimation Scheme for Wireless Sensor Networks
}

\author{
Radu Stoleru, John A. Stankovic \\ Department of Computer Science \\ University of Virginia \\ Charlottesville, VA 22903 \\ \{stoleru, stankovic@cs.virginia.edu\}
}

\begin{abstract}
Location information is of paramount importance for Wireless Sensor Networks (WSN). The accuracy of collected data can significantly be affected by an imprecise positioning of the event of interest. Despite the importance of location information, real system implementations, that do not use specialized hardware for localization purposes, have not been successful. In this paper, we propose a location estimation scheme that uses a probabilistic approach for estimating the location of a node in a sensor network. Our localization scheme makes use of additional knowledge of topology deployment. We assume a sensor network is deployed in a controlled manner, where the goal of the deployment is to form a grid topology. We evaluate our localization scheme through simulations, showing localization errors as low as $3 \%$ of radio range. We outperform similar localization schemes by obtaining $50 \%$ less error in localization, when compared to them. We also evaluate our localization solution and the DV-Hop scheme in a real system deployment, obtaining an average error in location of $79 \%$ of radio range, outperforming DV-Hop by approximately $40 \%$. We analyze the significant differences in performance between simulations and the real system deployment and stress the importance of further evaluations of real implementations. The result is an effective and realistic protocol that works in an actual system, under certain assumptions, because it exploits deployment information.
\end{abstract}

Keywords-wireless sensor network, location estimation, grid topology, probability.

\section{INTRODUCTION}

Recent advances in micro-electro-mechanical systems (MEMS) have triggered an enormous interest in wireless sensor networks (WSN). WSN are formed by large numbers of densely deployed nodes enabled with sensing and actuating capabilities.

These nodes have very limited processing and memory capabilities, limited energy resources and are mass produced, to reduce costs. Several challenging problems exist today in wireless sensor networks. Among those is the problem of obtaining location information by sensor nodes not equipped with specialized hardware (GPS, ultra-sound, acoustic, laser radiation). Due to the mostly static nature of a WSN, obtaining the location information by each sensor node is believed to be a one time or rare event. Adding hardware to each sensor node, to assist in the localization, is a costly solution, and, so far, it has been ruled out from mote-based system deployments.

The problem of localization in wireless sensor networks has been studied and evaluated mostly in simulators. Due to the severe hardware constraints imposed on wireless sensor nodes, real system implementations of the proposed simulated solutions have not produced encouraging results. Solutions that use the most tempting means of evaluating relative distances between sensor nodes - RF signal strength, have largely failed in practice, due to the unreliable nature and irregular pattern of the radio communication. We believe that solutions that are more robust to the randomness present in the radio communication require better calibration schemes together with localization schemes that are less sensitive to, sometimes, abnormal radio patterns. In this respect, to the best of our knowledge, we propose a first probabilistic algorithm for estimating location information in a sensor network. As a first step, we apply this solution to the case where the goal of topology deployment is a grid. This, in part, is fueled by our belief that a realistic deployment of a sensor network is not completely random, and an approximation to a uniform or even grid distribution can be expected. It is easy to envision methodologies for deployment of wireless sensor networks, from (un)manned aerial and terestrial vehicles, where the field of deployment is covered by parallel deployment paths and the sensor nodes are deployed at periodic intervals. Even for less regular deployment patterns, a much denser grid topology could be a good approximation. For our first investigation of a probabilistic approach to location estimation in sensor networks, we focus on sensor nodes deployed in a controlled manner, with the goal of forming a grid topology.

The main contributions of our paper are the following: we propose a localization scheme which is completely decentralized, it does not require special location or range finding infrastructure and the location accuracy is higher than comparable solutions. It accomplishes this by exploiting knowledge of the topology being targeted in the deployment. We also present experimental results of our scheme, obtained from a real implementation. Importantly, our scheme works in practice. We conclude our contributions by proposing further 
improvements to our solution, in order to tailor our approach to the realities imposed by real system deployments.

The remainder of the paper is organized as follows. The second section presents related work, identifies the localization schemes that inspired our solution and presents the distinctive characteristics that differentiate our work from previous research. In the third section, the Probability Grid localization algorithm is described. The performance evaluation of the proposed scheme is presented in section four. In section five we describe a system implementation on MICA2 motes and present the experimental results. We conclude in section six with a summary of the main contributions of this paper and present ideas for future research.

\section{RELATED WORK}

Several localization systems and algorithms have been proposed in the past. They ranged from solutions dependent on hardware support and the presence of an infrastructure set-up, to range-free solutions, where signal strength, hop count to known landmarks or apriori knowledge about the density of nodes in the network were used. In this section we present some of the proposed solutions and compare them with our scheme, pointing out the distinctive characteristics of our solution.

The Global Positioning System (GPS) [1] is well known today, widely used, both in military and civil applications. It has been developed over several decades and it relies on a constellation of satellites. Ranges to several satellites (by measuring the difference in the time of arrival of signals from different satellites) are used in a multilateration procedure to infer the position of the receiver. This localization scheme requires hardware that is both expensive and consumes significant power. Its use is limited to outdoor applications. Accuracy of a few meters is common in current commercial GPS devices, and even higher accuracy can be achieved in military applications. A practical and cost effective solution for localization in manually deployed sensor networks, solution that uses a GPS device attached to the deployer, is reported in [2]. The achieved localization error is on the order of 1 to 2 meters.

The RADAR system [3] uses the Received Signal Strength Indicator (RSSI) for determining the distance to known landmarks. In an offline phase, a centralized repository (database) of signal strengths at various positions with respect to a set of landmarks, is built. During the online phase, the best fit of transmitter's RSSI to the existing data in the repository, is used for obtaining a location. RADAR is capable of estimating the location of a mobile user to within a few meters.

The Cricket [4] location-support system is an example of a location scheme that uses a combination of radio and acoustic signals to estimate the distance to known anchors. Cricket can achieve a location granularity of 1.2 meters $x 1.2$ meters.

In comparison to the above mentioned localization scheme, our approach does not use ranging, i.e. it is range-free, does not require any infrastructure and it is completely decentralized.

Due to the costs and the inherent difficulties that the above solutions encounter when they are applied to adhoc wireless sensor networks, a different approach has been proposed and evaluated in the past. This approach is called range-free and it attempts to obtain location information from the proximity to a set of known landmarks. Representative for this approach are the localization schemes described below.

Bulusu et al. propose in [5] a localization scheme, called Centroid, in which each node localizes itself to the centroid of its proximate anchors. The advantages of this solution are its simplicity and ease of implementation. However, in this scheme, as well as in the next one, some nodes equipped with more powerful radios are assumed. In many real deployments of wireless sensor networks, it is difficult to envision the presence of sufficient and powerful enough anchors, to cover the entire network.

Along the same principles as found in the Centroid localization scheme, He et al. propose APIT [6], a scheme in which each node decides its position based on the possibility of being inside or outside of a triangle formed by any three anchors heard by the node. This solution, as Bulusu's, makes use of nodes with more powerful radios, and the difference between the RSSI received by the neighbors and itself to determine if a neighbor node is further or closer to all three anchors in each of the triangles. In a real deployment, calibration issues may arise, due to the significance placed on the RSSI value read by different sensor nodes.

A localization scheme that is also range-free and does not use more powerful anchors is the Global Coordinate System [7], developed at MIT. It uses apriori knowledge of the node density in the network, to estimate the average hop distance. The location accuracy obtained ranges from $8-10 \%$ of radio range when a subset of landmarks are hand-placed, and to 20$25 \%$ for the case in which the landmarks are randomly placed.

The localization solution with which our solution resembles the most, is the DV-Hop scheme, by Niculescu et al. at Rutgers [8]. The DV-Hop scheme uses the hop count from known landmarks to the nodes in the network to infer the distance. The protocol contains two phases. In the first phase, each node listens for possible beacons from anchors, propagated through flooding, and records the shortest hop count to each of the anchors. If a smaller hop count to an anchor is observed by a node, it is broadcast to its neighbors. The second phase of the protocol consists of the propagation of a correction factor, which represents an estimation of the true distance of one hop, as perceived by each anchor. This phase is initiated by each anchor, when beacons from other anchors are received.

An approach similar to the DV-family of localization schemes, but where special hardware is required, is AHLoS, proposed by Savides et al. [9]. AHLoS employs Time of Arrival (ToA) ranging techniques that require extensive hardware and solving relatively large nonlinear systems of 
equations. With its ultrasound hardware capabilities, positioning errors as small as 0.2 meters are reported.

\section{The ProbabILITy Grid Localization SCHEME}

In this section, we state the location estimation problem, our assumptions, and describe the algorithm for computing location information.

We consider a wireless sensor network, consisting of sensor nodes and anchor nodes. The anchor nodes represent only a small percentage and are either equipped with GPS or can acquire their location information through other means. No other assumptions, in terms of hardware capabilities, are made regarding the anchor nodes. In particular, anchor nodes do not have any increased communication range compared to other nodes. The remaining sensor nodes are unaware of their location. We assume a controlled deployment of the sensor nodes in the field, where the nodes are deployed in a grid topology, and where the unit length of the grid is known to all the nodes in the network. Our localization problem is to identify the correct position in the grid for each sensor node. Hence, the localization error may arise from an incorrect positioning in the grid of a sensor node, due to message loss, collisions and incorrect estimation of the Euclidian distance for one hop. We leave for future research, the investigation of an approximate grid deployment scenario, where we allow small errors in the true positioning of nodes around the vertexes of a grid. This error can be perceived as a second order perturbation to the actual location of a sensor node. Once its magnitude is obtained (a very difficult problem to solve), it could simply be added to the actual localization error that is obtained in the case of an exact grid deployment.

\section{A. The Probability Grid Localization Algorithm}

Let the deployment of the sensor nodes be in a grid topology of dimensions $\mathrm{M} \times \mathrm{N}$. Let $S$ be the set of all the nodes in the sensor network, and let $A$ be the set of all the anchors in the sensor network. Both sets, $S$ and $A$, are sets of ordered pairs (i,j) representing the grid points where the nodes are located.

For $\forall k \in S-A$, we define a hop-count vector $H^{k}$, that represents the hop count from each of the anchors in the set $A$ to the node ' $k$ ', as follows:

$$
H^{k}=\left(h_{1}^{k}, h_{2}^{k}, \ldots, h_{|\mathrm{A}|}^{k}\right)
$$

We represent the Probability Grid using an MxN matrix, as follows:

$$
F_{k}=\left(\begin{array}{cccc}
f_{11}^{k} & f_{12}^{k} & \ldots & f_{1 n}^{k} \\
f_{21}^{k} & f_{22}^{k} & \ldots & f_{2 n}^{k} \\
\ldots & \ldots & \ldots & \ldots \\
f_{m 1}^{k} & f_{m 2}^{k} & \ldots & f_{m n}^{k}
\end{array}\right), \forall k \in S-A
$$

where:

$f_{i j}^{k}=\left\{\begin{array}{cc}\text { probability of sensor node } k^{\prime} \text { to be at position }(i, j), & \text { if }(i, j) \notin A \\ 0, & \text { if }(i, j) \in A\end{array}\right.$

is the probability of sensor ' $k$ ' to be at the location (i,j).

Then, $f_{i j}^{k}$, an element of the Probability Grid matrix, can be expressed as follows:

$$
f_{i j}^{k}=\prod_{l=1}^{|A|} p_{i j}^{h_{l}^{k}},
$$

where $p_{i j}^{h_{l}^{k}}$ is the probability of node ' $k$ ', positioned at (i, j), to be $h_{l}^{k}$ hops from the $l$-th anchor.

One can observe that $p_{i j}^{h_{l}^{k}}$ is a discrete random variable that represents the number of hops for a particular Euclidian distance, in our case, between one anchor and the point of interest, i.e. (i, j).

The main features that the distribution function needs to exhibit are: to have two parameters (for our case, the distance between the node and one anchor - call it $\lambda$ and the number of hops existent between them - call it $\tau$ ), to be narrow and skewed positively for small values of $\lambda$ and become broader and relatively symmetric for larger values of $\lambda$. These requirements follow our intuition that for smaller values of the parameter $\lambda$, the number of hops $\tau$ has a limited range of possible values with higher and higher values being less and less probable (positively skewed). As the distance between the anchor and the node $(\lambda)$ increases the number of possibilities for the hop count $(\tau)$ increases and the distribution becomes bell-shaped, i.e. smaller and larger hop counts are equally probable. We have found through simulations that a Poisson distribution is a good approximation for our discrete random variable. The distribution is given by:

$$
P_{(\tau)}=\frac{\lambda^{\tau-1} e^{-\lambda}}{(\tau-1) !},
$$

where $\tau=1,2, \ldots$. is the number of hops and $\lambda$ is the distance, both between the anchor and the position being evaluated.

After computing the Probability Grid matrix, each node chooses the position $(i, j)$ in the grid for which the computed probability is maximum. Let $i_{\max }$ and $j_{\max }$ be the positions in 
the grid for node ' $k$ ' for which $f_{i_{\max } j_{\max }}^{k}=\max \left(f_{i j}^{k}\right), 1 \leq i \leq M, 1 \leq j \leq N$. The node ' $k$ ', will compute its location to be:

$\left(x_{k}, y_{k}\right)=\left(i_{\max } * G R I D \_U N I T_{-} S I Z E, j_{\max } * G R I D_{-} U N I T_{-} S I Z E\right)$

A walk-through our localization algorithm and the description of the localization protocol are presented in the following section.

\section{B. The Localization Scheme}

Our localization protocol is similar to the DV-Hop scheme, but it improves upon it by exploiting deployment information. The anchors flood the network with packets containing their IDs, their location and a hop count, initially set to zero. This flooding is either a global flooding, if the network size is small, or controlled flooding, if the size of the network is large and through local flooding all nodes are expected to hear from at least three anchors. During the flooding period, sensor nodes keep track of the shortest distance (number of hops) to each of the anchors they heard from. Once an anchor node receives beacons from several other anchors, an estimation for the Euclidian distance of one hop is calculated. This estimation is called a correction factor, and it is propagated in a second phase, through a controlled flooding. The correction factor computed by an anchor positioned at $\left(x_{i}, y_{i}\right)$ is given by the following equation [8]:

$$
C F_{i}=\frac{\sum_{j} \sqrt{\left(x_{i}-x_{j}\right)^{2}+\left(y_{i}-y_{j}\right)^{2}}}{\sum_{j} \tau_{j}}
$$

where $\tau_{j}$ is the number of hops between the current anchor, positioned at $\left(x_{i}, y_{i}\right)$, and the anchor positioned at $\left(x_{j}, y_{j}\right)$.

The first correction factor received by a sensor node is used in the estimation of the location. Subsequent correction factors are ignored. In this way, the correction factor is received only by the sensor nodes in the vicinity of the anchor.

Once a correction factor is received by a sensor node, the Probability Grid algorithm can be invoked. The information that is available at this time to each of the sensor nodes is the hop count to a set of anchors, the position of the anchors, the unit length of the grid and an estimate of the Euclidian distance of one radio hop. In our simulations we also assumed that the total size of the network was known. The dependency on this, however, can easily be removed. By using the position of at least three anchors and the unit length of the grid, a grid of an arbitrary size (i.e network size) can be constructed by each node. The size of the grid to be constructed can be parameterized, based on the density of anchors and nodes in the network. For higher densities of anchors in the network, smaller grid sizes are sufficient. Each sensor node evaluates the probability of being located at each of the grid points. For this, we first need to calculate $\lambda$ as follows:

$$
\lambda=\frac{\text { distance between grid point and anchor }}{\text { correction factor }}
$$

The computed $\lambda$ represents the distance, in hop count units, between the evaluated grid point and one anchor. Using the value of $\lambda$, and the actual hop count $h_{l}^{k}$, the probability $p_{i j}^{h_{l}^{k}}$ is calculated, as in (3). It represents the probability of node ' $k$ ' to be located at position $(\mathrm{i}, \mathrm{j})$ and to be $h_{l}^{k}$ hops from the $l$-th anchor.

Once the individual $p_{i j}^{h_{l}^{k}}$ factors are computed, the values of $f_{i j}^{k}$, elements of the Probability Grid matrix, given by (2) can be obtained. The next step is to complete the evaluation of all the elements of matrix $F_{k}$ as defined in (1).

The final location estimation position for node ' $k$ ', is obtained by identifying the maximum among the elements of matrix $F_{k}$. The indices $i_{\max }$ and $j_{\max }$ denoting the maximum element, are then applied to (4) and the estimated location $\left(x_{k}\right.$, $y_{k}$ ) for node ' $k$ ' is obtained.

\section{PERFORMANCE EVAlUATION}

In this section, we present our experimental results obtained through simulations using GloMoSim [10], a discrete-event simulator developed at UCLA. In our experiments we used the two-ray propagation pathloss model and IEEE 802.11 as the MAC layer protocol. Although the communication radius is circular and the links are symmetric, GloMoSim provides a fine-grained simulation, accounting for collisions, packet loss and energy consumption. The parameters of interest were: the ratio of anchors to the total number of nodes (anchors percentage), the size of the network (in number of hops), the density of nodes (this was varied by modifying the radio range) and the error in the location estimation of the anchors (anchor's location information was assumed to be imperfect). In all our simulations, except when simulating sensor networks with different sizes, we used 100 nodes deployed in a 10x10 grid topology on a square terrain with dimensions of 1000 meters x 1000 meters. When we evaluated networks with different sizes we used 36 nodes positioned in a 6x6 grid, 64 nodes positioned in a $8 \times 8$ grid and 100 nodes positioned in a 10x10 grid topology. Each experimental point was obtained from 300 simulations of networks with different anchor positions, positioned randomly. This ensured that the $90 \%$ confidence intervals are within $10 \%$ of the means. From our simulations we eliminated the scenarios where, even with a random placement of anchors, the positioning of the anchors was approximately in a straight line.

The most obvious metric to be used in estimating the effectiveness of a localization scheme is the localization error. We define the localization error by the following formula: 


$$
\text { error }=\sqrt{\left(x_{\text {est }}-x_{i}\right)^{2}+\left(y_{\text {est }}-y_{i}\right)^{2}} \text {, }
$$

where, $\left(x_{e s t}, y_{\text {est }}\right)$ is the estimated position of a node, located at $\left(x_{i}, y_{i}\right)$.

In our simulations we focused on identifying the effects of the aforementioned parameters, on the error in localization. Each of the following subsections details our experimental results when considering each of the parameters.

Due to the similarity of our approach to the Rutger's DVHop localization scheme we chose DV-Hop as the comparison benchmark. Because the pure version of the DV-Hop scheme does not make use of the additional knowledge gained from knowing that the deployment is attempting to create a grid topology, we implemented an enhanced version, called Grid DV-Hop, which attempts to place each node in a position in a grid closest to the actual computed location. The location with coordinates $\left(x_{\text {est }}^{\prime}, y^{\prime}\right.$ est $)$ estimated by the Grid DV-Hop is given by:

$$
\left(x_{e s t}^{\prime}, y_{e s t}^{\prime}\right)=\left(\left[x_{e s t}\right],\left[y_{e s t}\right]\right)
$$

where $\left(x_{\text {est }}, y_{\text {est }}\right)$ are the computed coordinates and the [.] is the nearest integer (in terms of grid units) function.

\section{A. Localization Error versus Anchors Percentage}

In this experiment we evaluate the effect that the number of anchors has on the location accuracy. Figure 1 depicts the effect on localization error when varying the percentage of anchors. The number of neighbors in this experiment was 8 . Networks with similar densities have been used in the evaluation of the DV-Hop family of localization schemes [8].

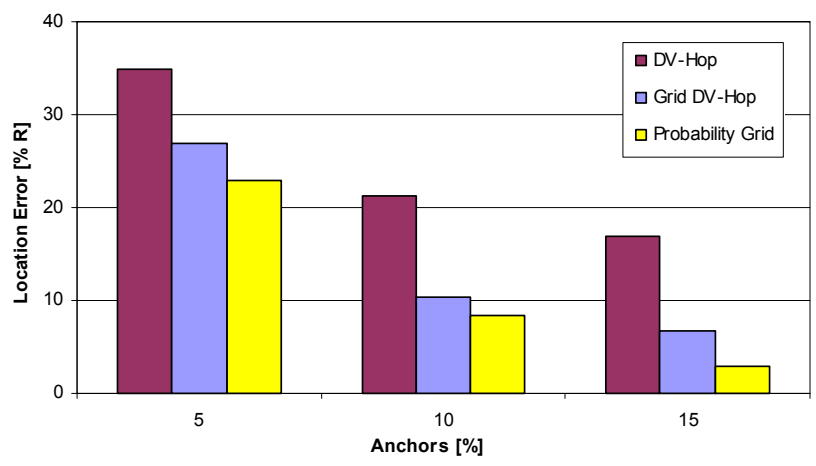

Figure 1. Location error for different ratios of anchors to total number of nodes (anchors percentages)

From Figure 1, it can be observed that the Probability Grid scheme outperforms the DV-Hop and Grid DV-Hop solutions, providing location errors that are smaller, on average, by $13 \%$ and $3 \%$ of radio range, respectively. When $15 \%$ of the nodes are anchors, the localization error for the Probability Grid is as low as $3 \%$.
It is interesting to note that the Probability Grid benefits more from an increased number of anchors, i.e. the error in localization decreases from about $23 \%$ to $3 \%$, where the DVHop's error in localization decreases from $36 \%$ to $17 \%$.

The reason for the improvements obtained in the Probability Grid scenario is due to the value present in the additional knowledge of deployment. When compared with pure DVHop, which does not assume a particular deployment topology, the differences are much larger than when using the Grid DVHop scheme, which uses the knowledge about the topology of deployment. Even with the Grid DV-Hop, sometimes the error in positioning is large enough that the estimation performed in equation (5) does not position the sensor node in the correct vertex on the grid.

\section{B. Localization Error versus Network Size}

In this experiment, we evaluate the effect the size of the network (in hops) has on the location error. We simulated three network sizes: $6 \times 6,8 \times 8$ and $10 \times 10$ grids, where the nodes were 100 meters apart. The number of neighbors for these simulations is also 8 , as in the previous one. The number of anchors were: 4, 7 and 10 for the $6 \times 6,8 \times 8$ and $10 \times 10$ respectively, aiming for approximately $10 \%$ anchors for each scenario. The results of the simulations are shown in Figure 2:

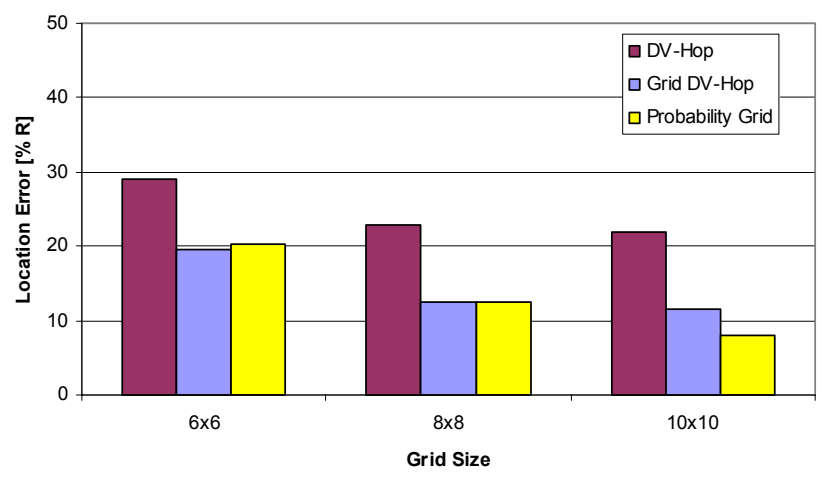

Figure 2. Location error for different network sizes

From our experiments, it can be observed that as the network size increases, the error in location estimation decreases for all three localization algorithms. The explanation is that the number of anchors used for estimating each position is increased, from 4 anchors, in the case of the 6x6 grid topology to 10 anchors in the case of 10x10 grid topology. All three localization schemes obtained improved localization accuracies, by approximately $10 \%$ of radio range.

\section{Localization Error versus Number of Neighbors}

In this experiment, we evaluate the effect the density of nodes has on the location error. The percentage of anchors was fixed to $15 \%$ for all scenarios. Figure 3 depicts the experimental results. It can be observed that for high densities, 
the error in localization increases. This is due to the fact that at higher densities, more sensor nodes are within one communication range and hence most likely they will have a similar hop count to multiple anchors, despite the fact that their positions differ.

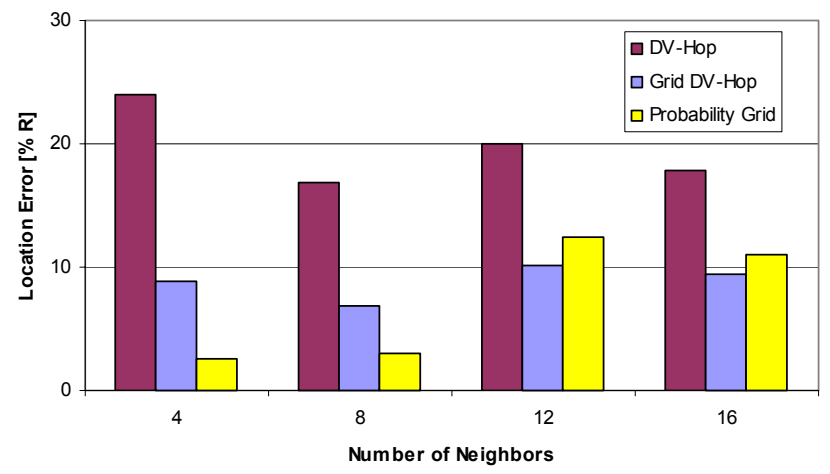

Figure 3. Location error for different node densities (number of neighbors)

We argue, however, that the increase in the location error can be reduced even in the presence of higher node densities, by a suitable reduction in the communication power, and implicitly in the communication range. This solution artificially reduces the density of nodes (per communication range) to values for which the hop count becomes a reasonable estimator of the distance between two points. The reduction of node density per communication range practically reduces the number of nodes that are within a specified number of hops from an anchor. Since the number of hops is the only metric to evaluate the distance between two points, then if a smaller the number of nodes (with different positions) have the same hop count with respect to an anchor, then a smaller average error is obtained.

\section{Localization Error versus Imprecision in Anchor Positioning}

In this experiment, we evaluated the effect that the error of the anchor's location has on the accuracy of location obtained by the rest of the nodes present in the network. The results are depicted in Figure 4. In the experiment, $10 \%$ of the sensor nodes were anchors and the number of neighbors was 8. From Figure 4, it can be observed that anchor positioning errors of up to $30 \%$ maintain an error in location of approximately $10 \%$ for the Probability Grid. Radio ranges of the most recent MICA2 sensor nodes vary from 15-30 meters. This translates into tolerable errors in the anchor position, that vary within 510 meters.

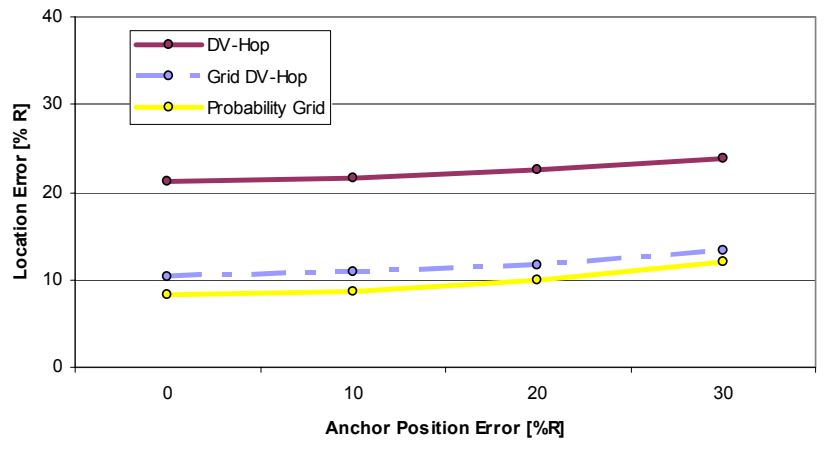

Figure 4. Location error for different anchor positioning errors

The obtained error in location is due to the incorrect positioning of the nodes in the grid topology. One extension to our localization scheme, which would incur a minor increase in the overhead, is, through additional beaconing, to have each node broadcast its location, and the confidence in its computation (probability). Nodes that potentially would position to the same location, with less confidence, would have to choose the next most probable position in the grid. We leave this enhancement, for future work.

\section{SYSTEM IMPLEMENTATION AND EVALUATION}

In this section we describe an implementation and an evaluation of our localization scheme. We compare the performance of our solution to that of DV-Hop. The implementation was done on MICA2 motes [11] from Berkeley. MICA2 platform uses a low power microcontroller, 8-bit Atmega128, and it has $128 \mathrm{~KB}$ of program FLASH memory, $4 \mathrm{~KB}$ of SRAM and $4 \mathrm{MB}$ external flash for data storage. Figure 5 shows a deployed MICA2 mote, in the field.

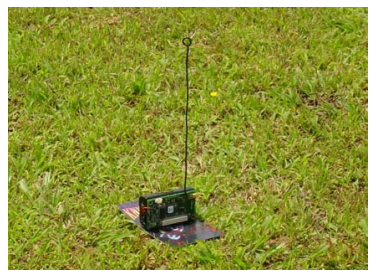

Figure 5. MICA2 sensor node placed in the field

The deployed system consisted of 25 motes, positioned in a $5 \times 5$ grid, approximately 12 meters apart. Three anchors were used and they were positioned at the following coordinates ( $\mathrm{x}$, y), in meters: $(12,0),(36,12)$ and $(24,48)$. Figure 6 , depicts the field of deployment and the approximate positioning of the sensor nodes in the field, shown with white dots. The field was relatively flat, with short cut grass and no metallic structures in the vicinity. 


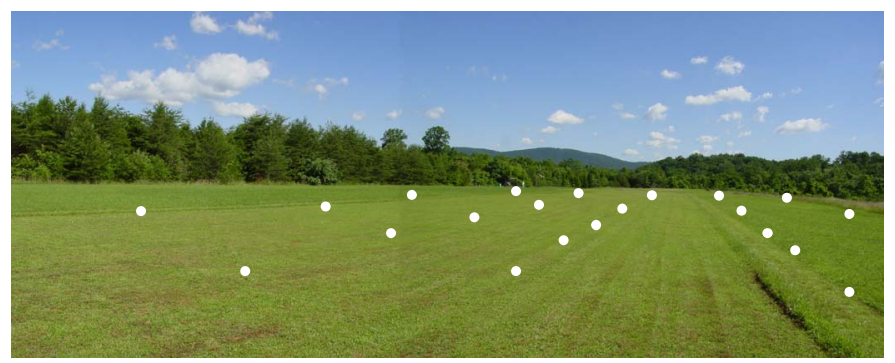

Figure 6. Field of deployment for the system

The system was deployed and went through the two phases of the algorithm: global flooding of anchor information (location and the number of hops between the receiving node and the anchor) and the correction propagation. The data (hop count to known anchors, the correction factor, the parent towards each anchor) was stored in flash memory. During the actual deployment, the DV-Hop localization scheme was applied. We used the data stored in the flash, offline, to evaluate the performance of our proposed Probability Grid localization scheme and the enhanced DV-Hop scheme, called Grid DV-Hop.

\section{A. Radio Calibration}

Several studies [12] [16] [17] have emphasized in the past the irregular and asymmetric nature of the radio communication in the sensor networks, and the importance of the calibration. The success of any solution that does not use hardware specialized for localization, and which relies on radio communication for inferring relative distances among sensor nodes, depends heavily on how good approximation to a circle, the actual radio range is. In addition, similarity in radio communication ranges has to exist among all the sensor nodes. In order to achieve these with some approximation, we took a simplistic approach, that involved a manual calibration of the radio for all the sensor nodes used in our experiment. More sophisticated calibration techniques have been reported [13] and their evaluation in real system deployments is very important.

It is important to note that we have not attempted to use the actual RSSI values for ranging. Our goal was to make the behavior of the radio more uniform, with some approximation, across sensor nodes. We did not use the RSSI values for ranging because it was shown in the past that the RSSI value does not constitute a reliable measure of distance between the transmitter and the receiver.

The calibration process involved three distinct steps, described below:

1) Receiver Calibration. In the first step, all sensor nodes were treated as receivers and calibrated against a single transmitter. We ensured that the RSSI for each node was in the same range $(-70 \mathrm{dBm}$ to $-90 \mathrm{dBm})$ for a particular fixed distance. For sensor nodes for which the RSSI was significantly different from the common range, a bias factor (representing the difference between the actual reading and the average of the range) was computed and used subsequently in RSSI measurements (added or subtracted from the actual reading). In Figure 7 we show a typical RSSI for one MICA2 mote, used in the experiment. Each point in the graph represents an average for 100 beacons, when the battery voltage was $2.9 \mathrm{~V}$. We have not observed drastic differences when battery voltage levels varied in the interval $[2.85 \mathrm{~V}$, $3.0 \mathrm{~V}]$. The experimental data was collected in the same field, and under the same conditions as in our localization experiments. For different MICA2 motes, the same pattern was observed, however the absolute values differed, hence the need for the bias correction. The bias factor is chosen such that at a distance of 15 meters, the RSSI value is $-80 \mathrm{dBm}$. This bias factor was stored in the flash memory and we observed, during experiments, that the radio characteristics remain approximately the same.

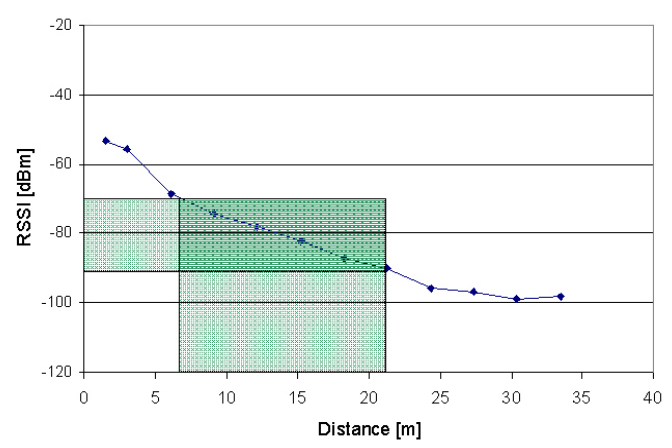

Figure 7. Radio signal strength versus distance

This first step of calibration ensured a relatively uniform behavior of the receivers.

2) Transmitter Calibration. The second phase of the calibration procedure consisted in calibrating all sensor nodes as transmitters, against a unique receiver. This was achieved by adjusting the sending power such that similar RSSI levels were observed at the receiver. Sensor nodes for which the sending power, even adjusted, could not be made large enough for the required level, were not included in the set of 25 motes used in the experiment.

3) Stable Hop Count Information Propagation. We have experimentally observed that it is possible that sometimes unusually long links were established in the network. Our observations are consistent with similar results reported in the literature [16]. In order to further decrease the influence that some of the longer radio links can have on the system, i.e. artificially obtaining very long one-hop links, we modified the mechanism by which the anchor information is flooded in the network. For this, each receiving node employed a two step process. In the first step, only packets with an RSSI within the interval [-90dBm, $-70 \mathrm{dBm}]$ were processed. We have observed more reliable, one hop links occurring in the aforementioned interval. Packets with RSSI values not falling in this interval were discarded. In the second step, a receiving sensor node 
deferred the decision regarding the hop count towards an anchor, until sufficiently stable information was present. For this, each sensor node maintained a moving average estimator of the RSSI value, with a window size of 10 , for each anchor and parent. A more complete analysis of various estimators of link reliability has been reported in [15]. Only after receiving at least 10 beacons and when the RSSI value for a particular parent was within $5 \%$ of the average, the hop count information was deemed reliable and it was further propagated in the network through flooding. Despite the overhead imposed by this reinforcement of hop count inference, we observed a quick convergence (approximately within 10 minutes, of a network of 25 motes) to stable hop counts towards the anchors, for all nodes in the network. Considering that the need for localization is generally limited to once per lifetime of the sensor network, the overhead imposed by the additional beaconing (for reinforcement of hop count information) is tolerable.

The main purpose for the aforementioned three phases of the calibration process was to enforce a limited communication range. This enforcement was necessary in order to prevent (as best as possible) abnormal patterns in radio communication, where unusually long links are established at the arrival of a packet with an extremely small signal strength at distances well beyond the regular radio communication range.

All the calibration parameters, namely the bias coefficient, the upper and lower bound on the permissible RSSI values, as well as the sending power level, were stored in the flash for subsequent evaluations. We have observed an acceptable consistency of RSSI and transmit power levels during the time period we evaluated the system. This consistency does not imply that the RSSI value is accurate enough for ranging techniques.

\section{B. Real System Evaluation Results}

The experimental results are summarized in Figure 8. For clarity we omit the Grid DV-Hop from Figure 8, but summarize the average location errors for all three localization schemes in Figure 9. The average location error for the DVHop scheme was $124 \%$ of the radio range, for the Grid DVHop the location error was \%117 of the radio range and for the Probability Grid scheme it was $79 \%$ of the radio range, where the radio range was approximately 15 meters.

In addition to the sources of errors in location estimation, that are present in simulations, the irregular radio pattern poses additional challenges in real system deployments.

In order to asses the performance of our proposed solution for localization, we simulated the same topology as the one used in the real implementation. The results are shown in Figure 9. It can be observed a major difference between the results produced in the simulator and the results obtained in the real system deployment. From simulations, the expected localization errors ranged from $15 \%$ to $50 \%$, for the Probability Grid and the DV-Hop schemes, respectively.
Experimental results positioned the error in location in the range $79 \%$ to $124 \%$. Even with our manual calibration process, in which we attempted to have a relatively uniform radio communication pattern, across sensor nodes, there remain differences between the simulator and real world. Analyzing the experimental data, we identified some pathological sensor nodes, that despite our calibration procedures, have produced much longer links than expected.

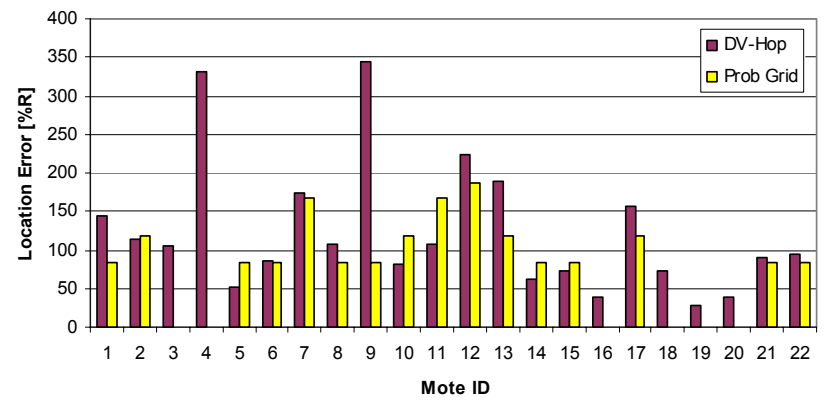

Figure 8. Location error for different nodes in a real system implementation

Our system evaluation showed that the Probability Grid location estimation scheme, outperforms DV-Hop related schemes in real deployments as well. A degradation in location estimation, of approximately $60 \%$ was observed in each of the investigated localization schemes.

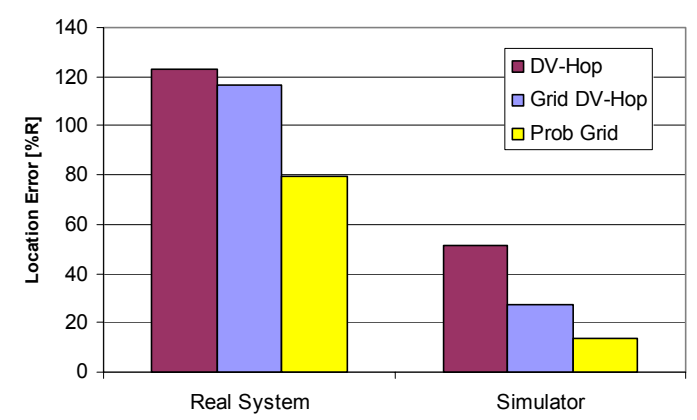

Figure 9. Location error, for different localization schemes, obtained in a real system implementation and simulator. An aggregate view

Our experimental results emphasize the importance of validation of localization schemes through real system implementations. Due to the hardware and cost constraints imposed on the existing wireless sensor nodes, simpler localization schemes need to rely only on the radio communication to infer proximity. However, through more robust calibration solutions and probabilistic localization algorithms, which are less sensitive to individual abnormal behavior, real implementations of localization procedures could produce more precise location estimation. Our proposed solution is a first step. 


\section{CONCLUSIONS AND FUTURE WORK}

In this paper we presented a localization scheme, called the Probability Grid, that can be used in WSN which have been deployed in a grid topology. Our scheme was inspired by a similar solution, called DV-Hop, from Rutgers [8], which uses hop count, from anchors to sensor nodes, as a measure of distance to known locations. The DV-Hop scheme is more general than our solution, since it does not use the knowledge about deployment topology. However, this generality does not help, since the errors are high in practical deployments. Probability Grid is more resistant than DV-Hop to situations when the hop count is not a very accurate measure of the distance between two points. This is due to the fact that in real deployments, the radio range is not circular. Previous research has shown the presence of long links, backward links or stragglers [16] and a significant deviation from a circular radio pattern [17]. Using a probabilistic approach, the Probability Grid considers the hop count for a particular distance to be a discrete random variable, that has a Poisson distribution.

Our solution is completely distributed and does not require special infrastructure. It only requires, as similar solutions do, to have a small percentage of the nodes, called anchors, aware of their position. We plan to extend our work in the following directions: a) empirically obtain a real distribution of hop counts for different distances. In simulations and limited experimental verification, a Poisson distribution proved satisfactory, however, from an empirically obtained distribution, we expect higher accuracy in a real system deployment; b) each node, through beaconing, can acquire additional information about its neighbors positions. This can serve as a reinforcement of accuracy of its own location computation and eliminate cases where multiple nodes localize themselves to the same position in the grid; c) employ local flooding from anchors to the adjacent nodes. We observed that the contribution made to the precision in position, made by anchors further away is much smaller than the contribution made by the anchors closer to the node; d) investigate the possibility of regionally centralizing the hop count information, in an attempt to better compute the optimal positioning of nodes in the grid, in order to decrease the total degree of uncertainty/entropy. A recent, entropy based approach for target localization is proposed by Wang et al. [18]. Their entropy-based sensor selection heuristic objective is to reduce the entropy of the target location distribution. Similarly, we plan on using the centralized approach in order to reduce the entropy of the sensor location distribution.

\section{ACKNOWLEDGMENT}

This work was supported by the DARPA IXO office, under the NEST project (grant number F336616-01-C-1905) and by the NSF grants CCR-0098269, CCR-0329609 and CCR0325197. The authors would like to thank the anonymous reviewers for their invaluable feedback.

\section{REFERENCES}

[1] B.W. Parkinson, J. Spilker, "Global Positioning System: theory and applications", in Progress in Aeronautics and Astronautics, vol. 163, 1996.

[2] R. Stoleru, T. He, J. A. Stankovic, "Walking GPS: A practical solution for localization in manually deployed wireless sensor networks", $1^{\text {st }}$ IEEE Workshop on Embedded Networked Sensors (EmNetS), Tampa, Florida, 2004

[3] P. Bahl, V.N. Padmanabhan, "RADAR: An in-building RFbased user location and tracking system", in Proceedings of IEEE Infocom, Tel Aviv, Israel, 2000.

[4] N. Pryiantha, A. Chakaborty, H. Balakrishnan, "The Cricket location-support system", in Proceedings of ACM MobiComm 2000, pages 32-43, Boston, Massachussetts, 2000.

[5] N. Bulusu, J. Heidemann, D. Estrin, "GPS-less low cost outdoor localization for very small devices", in IEEE Personal Communications Magazine, Special Issue on Smart Spaces and Environments, 2000

[6] T. He, C. Huang, B. Blum, J.A. Stankovic, T. Abdelzaher, "Range-Free localization schemes for large scale sensor networks" in The Ninth Annual International Conference on Mobile Computing and Networking, San Diego, CA, September 2003.

[7] R. Nagpal, H. Shrobe, J. Bachrach, "Organizing a global coordinate system for local information on an ad hoc sensor network", in A.I Memo 1666. MIT A.I. Laboratory, August 1999.

[8] D. Niculescu, B. Nath, "DV-based positioning in adhoc networks" in Telecommunication Systems, pages 267-280, Kluwer Academic Publishers, 2003.

[9] A. Savides, C. Han, M. Srivastava, "Dynamic fine-grained localization in adhoc networks of sensors", in Proceedings of ACM MobiComm 2001, Rome, Italy, 2001.

[10] X. Zheng, R. Bragodia, M. Gerla, "GloMoSim: a library for parallel simulations of large-scale wireless networks", in Proceedings of the 12th Workshop on Parallel and Distributed Simulations - PADS '98, May, 1998.

[11] http://www.xbow.com/Products/Product_pdf_files/Wireless_pdf/ 6020-0042-05_A_MICA2.pdf

[12] A. Woo, T. Tong, D. Culler, "Taming the underlying challenges of reliable multihop routing in sensor networks", in Sensys 2003, Los Angeles, October, 2003

[13] K. Whitehouse, D. Culler, "Calibration as parameter estimation in sensor networks", in First ACM International Workshop on Wireless Sensor Networks and Applications, Atlanta, GA, September 2002.

[14] A. Cerpa, J.L. Wong, L. Kuang, M. Potkonjak, D. Estrin, "Statistical model of lossy links in wireless sensor networks", CENS Technical Report, April 2004

[15] A. Woo, D. Culler, "Evaluation of efficient link reliability estimators for low-power wireless networks", UCB Technical Report, November 2003

[16] D. Ganesan, B. Krishnamachari, A. Woo, D. Culler, D. Estrin, S. Wicker, "Complex behavior at scale: an experimental study of low power wireless sensor networks", Technical Report, UCLA/CSD-TR 02-0013

[17] G. Zhou, T. He, S. Krishnamurthy, J.A. Stankovic, "Impact of radio asymmetry on wireless sensor networks", in MobiSys 2004, Boston, MA, June 2004.

[18] H. Wang, K. Yao, G. Pottie, D. Estrin, "Entropy-based sensor selection heuristic for target localization", in Proceedings of $3^{\text {rd }}$ International Symposium on Information Processing in Sensor Networks IPSN, Berkeley, April 2004 